hep-th/0203209

March 2002

\title{
Geometrical Construction of Heterogeneous Loop Amplitudes in 2D Gravity
}

\author{
Masahiro ANAZAWA and Atushi ISHIKAWA f \\ $\dagger$ Tohoku Institute of Technology, Sendai 982-8577, Japan \\ $\ddagger$ Kanazawa Gakuin University, Kanazawa 920-1392, Japan
}

\begin{abstract}
We study a disk amplitude which has a complicated heterogeneous matter configuration on the boundary in a system of the $(3,4)$ conformal matter coupled to two-dimensional gravity. It is analyzed using the two-matrix chain model in the large $N$ limit. We show that the disk amplitude calculated by Schwinger-Dyson equations can completely be reproduced through purely geometrical consideration. From this result, we speculate that all heterogeneous loop amplitudes can be derived from the geometrical consideration and the consistency among relevant amplitudes.
\end{abstract}

\footnotetext{
${ }^{\dagger}$ E-mail address: anazawa@tohtech.ac.jp

₹ E-mail address: ishikawa@kanazawa-gu.ac.jp
} 


\section{Introduction}

The $(m, m+1)$ unitary minimal conformal model coupled to two-dimensional gravity can be analyzed by the two-matrix chain model or the multi-matrix chain model [1]-5] under appropriate scaling limits. In two-dimensional gravity, loop amplitude is one of the most important physical quantities, which is the amplitude for a surface with loop-like boundaries. The $(m, m+1)$ minimal model has $m-1$ matter states [6], so in general, we have to deal with loops on which matter configurations are not restricted to homogeneous ones [7]- [12].

In Refs. [11, 12], some disk amplitudes whose boundaries have heterogeneous matter states are studied in the case of the tricritical Ising model ( $m=4$ case). It is found that the obtained amplitudes have simple geometrical interpretation. The heterogeneous loops make interesting splitting and sticking interaction regularly. Here a question arises. Can we decide any heterogeneous loop amplitudes only from the geometrical consideration? In other words, does the geometrical consideration give enough information to decide any heterogeneous loop amplitudes?

In order to address this question, in this paper, we study a disk amplitude with rather complicated boundary condition in the case of the Ising model ( $m=3$ case). We show that the amplitude calculated by Schwinger-Dyson equations can be reproduced through purely geometrical consideration. We speculate that any heterogeneous loop amplitudes can be decided geometrically.

\section{Amplitude from Schwinger-Dyson equations}

In this section, we examine a disk amplitude with heterogeneous matter boundary condition using Schwinger-Dyson equations. Let us start with the action of the two-matrix model

$$
S(A, B)=\frac{N}{\Lambda} \operatorname{tr}\{U(A)+U(B)-A B\} .
$$

Here $A$ and $B$ are $N \times N$ unitary matrix variables, and $\Lambda$ is the bare cosmological constant. As a critical potential which realizes the Ising model coupled to two-dimensional gravity, we take

$$
U(\phi)=\alpha \phi+\frac{\beta}{2} \phi^{2}+\frac{\gamma}{3} \phi^{3}, \quad(\alpha, \beta, \gamma)=(3,-3,-1) .
$$

This can be found using the orthogonal polynomial method [5, 11].

In this paper we will focus on the amplitude

$$
W_{A B A B}\left(p_{1}, q_{1}, p_{2}, q_{2}\right)=\sum_{k, l, m, n=0}^{\infty} \frac{\Lambda}{N}\left\langle\operatorname{tr}\left(A^{k} B^{l} A^{m} B^{n}\right)\right\rangle p_{1}^{-k-1} q_{1}^{-l-1} p_{2}^{-m-1} q_{2}^{-n-1}
$$

and its continuum universal counterpart $w_{A B A B}\left(\zeta_{A 1}, \zeta_{B 1}, \zeta_{A 2}, \zeta_{B 2}, t\right)$ in the large $N$ limit. Here $p_{i}$ and $q_{i}$ are bare boundary cosmological constants, $\zeta_{A_{i}}$ and $\zeta_{B_{i}}$ are their renormalized counterparts, and $t$ is the renormalized cosmological constant. The insertion of the operator $\operatorname{tr}\left(A^{k} B^{l} A^{m} B^{n}\right)$ 
makes a loop-like boundary on which a part with spins up and a part with spins down appear by turns. In the large $N$ limit, the corresponding continuum amplitude becomes the disk amplitude whose boundary consists of four arcs with distinct matter states.

Let us start with the Schwinger-Dyson equation

$$
0=\sum_{a} \int d A d B \frac{\partial}{\partial A^{a}}\left\{\operatorname{tr}\left(A^{k} t^{a} B^{l} A^{m} B^{n}\right) e^{-S(A, B)}\right\}
$$

here the hermitian matrix $A$ is expressed as $A=\sum_{a} A^{a} t^{a}$ using the bases of hermitian matrices $\left\{t^{a}\right\}$. Rewriting Eq. (2.4) explicitly, we obtain

$$
\begin{aligned}
0= & \sum_{i=0}^{k-1}\left[A^{i}\right]\left[A^{k-i-1} B^{l} A^{m} B^{n}\right]+\sum_{i=0}^{m-1}\left[B^{l} A^{m-i-1}\right]\left[A^{k} A^{i} B^{n}\right]-\alpha\left[A^{k} B^{l} A^{m} B^{n}\right] \\
& -\beta\left[A^{k+1} B^{l} A^{m} B^{n}\right]-\gamma\left[A^{k+2} B^{l} A^{m} B^{n}\right]+\left[A^{k} B^{l+1} A^{m} B^{n}\right]
\end{aligned}
$$

here we use the notation $\left[A^{k} B^{l} \cdots\right]=\frac{\Lambda}{N}\left\langle\operatorname{tr}\left(A^{k} B^{l} \cdots\right)\right\rangle$. Multiplying $p_{1}^{-k-1} q_{1}^{-l-1} p_{2}^{-m-1} q_{2}^{-n-1}$ and summing up with respect to $k, l, m$ and $n$ indices, we obtain the equation in terms of resolvent amplitudes

$$
\begin{aligned}
0= & \left(W_{A}\left(p_{1}\right)-v\left(p_{1}\right)+q_{1}\right) W_{A B A B}\left(p_{1}, q_{1}, p_{2}, q_{2}\right)+\left(W_{B A}\left(q_{1}, p_{2}\right)-1\right) W_{A A B}\left(p_{1}, p_{2}, q_{2}\right) \\
& +\left(\beta+\gamma p_{1}\right) W_{B A B}\left(q_{1}, p_{2}, q_{2}\right)+\gamma W^{(A)}{ }_{B A B}\left(q_{1}, p_{2}, q_{2}\right) .
\end{aligned}
$$

In Eq. (2.6) and in the following, we use the notations $v(p)=\alpha+\beta p+\gamma p^{2}$,

$$
\begin{gathered}
W_{M_{1} M_{2} \ldots\left(r_{1}, r_{2}, \cdots\right)}=\sum_{k, l, \cdots=0}^{\infty} \frac{\Lambda}{N}\left\langle\operatorname{tr}\left(M_{1}^{k} M_{2}^{l} \cdots\right)\right\rangle r_{1}^{-k-1} r_{2}^{-l-1} \cdots, \\
W^{(M)} M_{1}{ }^{\left(M^{\prime}\right)} M_{2} \ldots\left(r_{1}, r_{2}, \cdots\right)=\sum_{k, l, \cdots=0}^{\infty} \frac{\Lambda}{N}\left\langle\operatorname{tr}\left(M M_{1}^{k} M^{\prime} M_{2}^{l} \cdots\right)\right\rangle r_{1}^{-k-1} r_{2}^{-l-1} \cdots,
\end{gathered}
$$

where $M, M^{\prime}$ and $M_{i}$ denote the matrices $A$ or $B$. Using the relations $W_{B A}\left(q_{1}, p_{2}\right)=W_{A B}\left(p_{2}, q_{1}\right)$ and $W_{B A B}\left(q_{1}, p_{2}, q_{2}\right)=W_{A A B}\left(q_{2}, q_{1}, p_{2}\right)$, we observe that $W_{A B A B}$ can be expressed in terms of $W_{A}, W_{A B}, W_{A A B}$ and $W_{B A B}^{(A)}$.

As for $W_{B A B}^{(A)}$, from the resolvent expression for the $l=0$ case of Eq. (2.5) and the relations $W_{A B}^{(A)}\left(p_{2}, q_{2}\right)=p_{2} W_{A B}\left(p_{2}, q_{2}\right)-W_{B}\left(q_{2}\right)$ and $W_{A}^{(B)}{ }_{A B}\left(p_{1}, p_{2}, q_{2}\right)=W_{B A B}^{(A)}\left(p_{2}, q_{2}, p_{1}\right)$, we obtain

$$
\begin{aligned}
W_{B A B}^{(A)}\left(p_{2}, q_{2}, p_{1}\right)= & -\left(W_{A}\left(p_{1}\right)+W_{A}\left(p_{2}\right)-v\left(p_{1}\right)\right) W_{A A B}\left(p_{1}, p_{2}, q_{2}\right) \\
& -\left(\beta+\gamma\left(p_{1}+p_{2}\right)\right) W_{A B}\left(p_{2}, q_{2}\right)+\gamma W_{B}\left(q_{2}\right) .
\end{aligned}
$$

Combining Eqs. (2.6) and (2.9), we find that $W_{A B A B}$ can be expressed in terms of $W_{A}, W_{B}$, $W_{A B}$ and $W_{A A B}$. Furthermore using the relations

$$
W_{A A B}\left(p_{1}, p_{2}, q\right)=-\frac{W_{A B}\left(p_{1}, q\right)-W_{A B}\left(p_{2}, q\right)}{p_{1}-p_{2}},
$$




$$
\begin{aligned}
& W_{A B}(p, q)=\frac{W_{A}(p)-(\beta+\gamma p) W_{B}(q)-\gamma W_{B}{ }^{(A)}(q)}{W_{A}(p)-v(p)+q}, \\
& W_{B}^{(A)}(q)=-\left(W_{B}(q)-v(q)\right) W_{B}(q)-(\beta+\gamma q) \Lambda-\gamma[A],
\end{aligned}
$$

we find, in the end, that $W_{A B A B}$ can be expressed in terms of $W_{A}$ and $W_{B}$. (Here the last two equations are obtained in a similar way to Refs. [7, 8, 10, 11, 12].)

The homogeneous disk amplitudes $W_{A}$ and $W_{B}$ satisfy a third order equation. They are expanded as

$$
W_{A}(p)=W_{B}(p)=3-2 a \zeta+\left(\frac{a}{2}\right)^{4 / 3} w(\zeta)+\mathcal{O}\left(a^{5 / 3}\right)
$$

here $a$ is the lattice spacing and

$$
w(\zeta)=\left(\zeta+\sqrt{\zeta^{2}-t}\right)^{4 / 3}+\left(\zeta-\sqrt{\zeta^{2}-t}\right)^{4 / 3}
$$

is the continuum homogeneous disk amplitude [13] under the renormalization $\Lambda=10-a^{2} t$ and $p=a \zeta$. This amplitude satisfies the third order equation

$$
w(\zeta)^{3}-3 t^{4 / 3} w(\zeta)-16 \zeta^{4}+16 t \zeta^{2}-2 t^{2}=0
$$

Combining all of the above equations, we obtain the continuum heterogeneous disk amplitude $w_{A B A B}$. From the explicit calculation, we find that terms in $\mathcal{O}\left(a^{5 / 3}\right)$ in Eq. 2.13) do not affect the calculation of $w_{A B A B}$, and $w_{A B A B}$ is expressed only in terms of $w\left(\zeta_{A_{i}}\right)$ and $w\left(\zeta_{B_{j}}\right)$. From the $a^{1}$ order terms of $16 W_{A B A B}$, we obtain the continuum disk amplitude $w_{A B A B}$ as follows:

$$
\begin{aligned}
w_{A B A B}\left(\zeta_{A_{1}}, \zeta_{B_{1}}, \zeta_{A_{2}}, \zeta_{B_{2}}\right) & \\
= & -\frac{w\left(\zeta_{A_{1}}\right)+w\left(\zeta_{A_{2}}\right)}{\zeta_{A_{1}}-\zeta_{A_{2}}}\left(\frac{w\left(\zeta_{A_{1}}\right)^{2}}{\left(\zeta_{A_{1}}+\zeta_{B_{1}}\right)\left(\zeta_{A_{1}}+\zeta_{B_{2}}\right)}-\frac{w\left(\zeta_{A_{2}}\right)^{2}}{\left(\zeta_{A_{2}}+\zeta_{B_{1}}\right)\left(\zeta_{A_{2}}+\zeta_{B_{2}}\right)}\right) \\
& -\frac{w\left(\zeta_{B_{1}}\right)+w\left(\zeta_{B_{2}}\right)}{\zeta_{A_{1}}-\zeta_{A_{2}}}\left(\frac{w\left(\zeta_{A_{1}}\right)^{2}}{\left(\zeta_{A_{1}}+\zeta_{B_{1}}\right)\left(\zeta_{A_{1}}+\zeta_{B_{2}}\right)}-\frac{w\left(\zeta_{A_{2}}\right)^{2}}{\left(\zeta_{A_{2}}+\zeta_{B_{1}}\right)\left(\zeta_{A_{2}}+\zeta_{B_{2}}\right)}\right) \\
& -\frac{w\left(\zeta_{B_{1}}\right) w\left(\zeta_{B_{2}}\right)}{\zeta_{A_{1}}-\zeta_{A_{2}}}\left(\frac{w\left(\zeta_{A_{1}}\right)}{\left(\zeta_{A_{1}}+\zeta_{B_{1}}\right)\left(\zeta_{A_{1}}+\zeta_{B_{2}}\right)}-\frac{w\left(\zeta_{A_{2}}\right)}{\left(\zeta_{A_{2}}+\zeta_{B_{1}}\right)\left(\zeta_{A_{2}}+\zeta_{B_{2}}\right)}\right) \\
& -3 t^{4 / 3}\left(w\left(\zeta_{A_{1}}\right)+w\left(\zeta_{A_{2}}\right)\right) \frac{\zeta_{A_{1}}+\zeta_{A_{2}}+\zeta_{B_{1}}+\zeta_{B_{2}}}{\left(\zeta_{A_{1}}+\zeta_{B_{1}}\right)\left(\zeta_{A_{2}}+\zeta_{B_{1}}\right)\left(\zeta_{A_{1}}+\zeta_{B_{2}}\right)\left(\zeta_{A_{2}}+\zeta_{B_{2}}\right)} \\
& +\left(A_{1} \leftrightarrow B_{1}, A_{2} \leftrightarrow B_{2}\right) .
\end{aligned}
$$

Here the last expression $\left(A_{1} \leftrightarrow B_{1}, A_{2} \leftrightarrow B_{2}\right)$ denotes the terms which are obtained by interchanging $A_{i}$ and $B_{i}$ in all of the preceding terms. As the continuum part, we have taken terms which are non-analytic in all of the variables $\zeta_{A_{i}}, \zeta_{B_{j}}$ and $t$. We should notice that the form of the amplitude (2.16) is not unique because of the identity equation (2.15). This uncertainty, however, does not arise in the geometrical consideration in the next section. 


\section{Amplitude from geometrical consideration}

In this section, we will show that the heterogeneous disk amplitude $w_{A B A B}$ obtained in section 2 can be reproduced through purely geometrical consideration.

At first let us briefly review the mechanism of the interaction of heterogeneous boundary in the case of $w_{A B}$ [11], which is the continuum counterpart of $W_{A B}$. In this case, the boundary loop consists of two parts on which the spin state is up or down. According to boundary conformal field theory, discontinuity in the matter boundary conditions corresponds to the insertion of a boundary operator in Refs. [14, 9]. So in the case of $w_{A B}$, we consider that two spin operators $\phi_{+-} \equiv \sigma$ are situated at the points where spin state changes (see Fig. 1). The continuum amplitude $w_{A B}$ is calculated to be [9, 10]

$$
w_{A B}\left(\zeta_{A}, \zeta_{B}\right)=\frac{w\left(\zeta_{A}\right)^{2}+w\left(\zeta_{B}\right)^{2}+w\left(\zeta_{A}\right) w\left(\zeta_{B}\right)-3 t^{4 / 3}}{\zeta_{A}+\zeta_{B}} .
$$

By performing the inverse Laplace transformation, we obtain the disk amplitude $\mathcal{W}_{A B}\left(\ell_{A}, \ell_{B}\right)$ in terms of the lengths of the boundaries (see appendix A),

$$
\begin{aligned}
\mathcal{W}_{A B}\left(\ell_{A}, \ell_{B}\right)= & \mathcal{L}_{\mathcal{A}}{ }^{-1} \mathcal{L}_{\mathcal{B}}{ }^{-1}\left[w_{A B}\left(\zeta_{A}, \zeta_{B}\right)\right] \\
= & \theta\left(\ell_{A}-\ell_{B}\right)(\mathcal{W} * \mathcal{W})\left(\ell_{A}-\ell_{B}\right)+\theta\left(\ell_{B}-\ell_{A}\right)(\mathcal{W} * \mathcal{W})\left(\ell_{B}-\ell_{A}\right) \\
& +\int_{0}^{\min \left(\ell_{A}, \ell_{B}\right)} d \ell \mathcal{W}\left(\ell_{A}-\ell\right) \mathcal{W}\left(\ell_{B}-\ell\right)-3 t^{4 / 3} \delta\left(\ell_{A}-\ell_{B}\right) .
\end{aligned}
$$

From Eq. (3.2) we find that the following geometrical interpretation is possible [1]. The original heterogeneous loop splits into two homogeneous loops. The first three terms in Eq. (3.2) correspond to the three diagrams in Fig. 2 respectively. This loop splitting phenomenon can be summarized in the following rules.

1. Boundary A 1 sticks to boundary B so that a heterogeneous loop splits into homogeneous loops. The split disks are connected by the double line which is formed by sticking of boundaries A and B.

2. The spin operator $\sigma$ is situated at each end of the double line.

The spin operator $\sigma$ separates the boundary into two sides. One side forms homogeneous looplike boundary and the other forms a stuck double line. We speculate that the spin operator $\sigma$ has the effect to make discontinuity in the geometrical state of boundary as well as in the matter state.

Now let us derive $w_{A B A B}$ through a purely geometrical consideration. For simplicity, we will neglect the terms proportional to $t^{4 / 3}$ in Eqs. (2.16) and (3.1), because they only make

\footnotetext{
${ }^{1}$ We refer to the part of boundary on which the spin state is up as boundary A etc, in the same way to [11].
} 
the argument complicate. We can deal with these terms in a similar manner. The rules of the splitting phenomenon should equally be applied in this case. We will employ the rules and sum up all possible terms. Here the weights of the respective terms, however, are not fixed. The relative weights will be fixed by requiring the consistency with $w_{A B}$ and the expected symmetries of the amplitude $w_{A B A B}$. The resultant amplitude will completely coincide with that derived from the Schwinger-Dyson equations up to overall normalization.

In the following we will explain how to derive the amplitude $w_{A B A B}$ in detail. In the case of $w_{A B A B}$, there are four spin operators $\sigma$ on the loop-like boundary. Let us focus on one of them (denoted by $\circ$ in Fig. 3). After loop splitting, the spin operator we focus is in touch with another part of the boundary. According to the point with which it is in touch, there are four possibilities, (a), (b), (c) and (d) in Fig. 3. As an example let us consider the case (a) concretely. The spin operator we focus is at one end of the double line (see Fig. 团). At the other end of the double line, one of the remaining spin operators will be situated. According to the spin operator situated there, the case (a) is classified into three sub cases, (a-1), (a-2) and (a-3) in Fig. 周. Then the amplitude corresponding to the case (a) is written as follows (see appendix A):

$$
\begin{aligned}
\mathcal{G}_{a} & =a_{1} \frac{w_{A A B}\left(\zeta_{A_{1}}, \zeta_{A_{2}}, \zeta_{B_{1}}\right) w\left(\zeta_{B_{2}}\right)}{\zeta_{A_{2}}+\zeta_{B_{2}}}+a_{2} \frac{w_{B B A}\left(\zeta_{B_{1}}, \zeta_{B_{2}}, \zeta_{A_{1}}\right) w\left(\zeta_{B_{2}}\right)}{\zeta_{A_{2}}+\zeta_{B_{2}}} \\
& +\frac{\left\{a_{3} w\left(\zeta_{A_{1}}\right) w\left(\zeta_{A_{2}}\right)+a_{4} w\left(\zeta_{A_{1}}\right) w\left(\zeta_{B_{1}}\right)+a_{5} w\left(\zeta_{B_{2}}\right) w\left(\zeta_{A_{2}}\right)+a_{6} w\left(\zeta_{B_{2}}\right) w\left(\zeta_{B_{1}}\right)\right\} w\left(\zeta_{B_{2}}\right)}{\left(\zeta_{A_{1}}+\zeta_{B_{2}}\right)\left(\zeta_{A_{2}}+\zeta_{B_{1}}\right)\left(\zeta_{A_{2}}+\zeta_{B_{2}}\right)} .
\end{aligned}
$$

Here $a_{i}$ represent unknown weight constants, which will be determined later, and $w_{A A B}$ and $w_{B B A}$ represent the continuum counterparts of the amplitudes $W_{A A B}$ and $W_{B B A}$ respectively. Similarly the case (b) is classified into two sub cases, (b-1) and (b-2) (see Fig. (6). The corresponding amplitude is

$$
\mathcal{G}_{b}=b_{1} \frac{w_{A A B}\left(\zeta_{A_{1}}, \zeta_{A_{2}}, \zeta_{B_{1}}\right) w\left(\zeta_{B_{2}}\right)}{\zeta_{A_{1}}+\zeta_{B_{2}}}+b_{2} \frac{w_{A A B}\left(\zeta_{A_{1}}, \zeta_{A_{2}}, \zeta_{B_{1}}\right) w\left(\zeta_{A_{1}}\right)}{\zeta_{A_{1}}+\zeta_{B_{2}}}
$$

where $b_{i}$ are unknown weight constants. The cases (c) and (d) are obtained by interchanging $A_{i}$ with $B_{i}$ in the cases (b) and (a) respectively:

$$
\mathcal{G}_{c}=\left.\mathcal{G}_{b}\right|_{A \leftrightarrow B}, \quad \mathcal{G}_{d}=\left.\mathcal{G}_{a}\right|_{A \leftrightarrow B} .
$$

In the cases (b) and (c), we have doubly counted several terms. The next amplitude corresponds to the doubly counted terms,

$$
\begin{aligned}
\mathcal{G}_{e} & =-b_{1} \frac{w\left(\zeta_{A_{2}}\right) w\left(\zeta_{B_{2}}\right)\left(w\left(\zeta_{A_{1}}\right)+w\left(\zeta_{B_{1}}\right)\right)}{\left(\zeta_{A_{1}}+\zeta_{B_{1}}\right)\left(\zeta_{A_{1}}+\zeta_{B_{2}}\right)\left(\zeta_{A_{2}}+\zeta_{B_{1}}\right)}-b_{2} \frac{w\left(\zeta_{A_{1}}\right) w\left(\zeta_{B_{1}}\right)\left(w\left(\zeta_{A_{1}}\right)+w\left(\zeta_{B_{1}}\right)\right)}{\left(\zeta_{A_{1}}+\zeta_{B_{1}}\right)\left(\zeta_{A_{1}}+\zeta_{B_{2}}\right)\left(\zeta_{A_{2}}+\zeta_{B_{1}}\right)} \\
& +\frac{w\left(\zeta_{B_{1}}\right) w\left(\zeta_{B_{2}}\right)\left(e_{1} w\left(\zeta_{A_{1}}\right)+e_{2} w\left(\zeta_{B_{1}}\right)\right)}{\left(\zeta_{A_{1}}+\zeta_{B_{1}}\right)\left(\zeta_{A_{1}}+\zeta_{B_{2}}\right)\left(\zeta_{A_{2}}+\zeta_{B_{1}}\right)}+\frac{w\left(\zeta_{A_{1}}\right) w\left(\zeta_{A_{2}}\right)\left(e_{1} w\left(\zeta_{B_{1}}\right)+e_{2} w\left(\zeta_{A_{1}}\right)\right)}{\left(\zeta_{A_{1}}+\zeta_{B_{1}}\right)\left(\zeta_{A_{1}}+\zeta_{B_{2}}\right)\left(\zeta_{A_{2}}+\zeta_{B_{1}}\right)} .
\end{aligned}
$$


Here the first term comes from the double counting between the cases (b-1) and (c-1) (see Fig. đ). The second term comes from that between the cases (b-2) and (c-2) (see Fig. 8). The third term represents that between the cases (b-1) and (c-2) (see Fig. 9). The last term represents that between the cases (b-2) and (c-1) (see Fig. 10).

The amplitude we aim at is obtained by summing up all the cases

$$
\mathcal{G}=\mathcal{G}_{a}+\mathcal{G}_{b}+\mathcal{G}_{c}+\mathcal{G}_{d}+\mathcal{G}_{e}
$$

By using the relations

$$
\begin{gathered}
w_{A A B}\left(\zeta_{1}, \zeta_{2}, \xi\right)=w_{B B A}\left(\zeta_{1}, \zeta_{2}, \xi\right)=-\frac{w_{A B}\left(\zeta_{1}, \xi\right)-w_{A B}\left(\zeta_{2}, \xi\right)}{\zeta_{1}-\zeta_{2}} \\
w_{A B}(\zeta, \xi)=\frac{w(\zeta)^{2}+w(\xi)^{2}+w(\zeta) w(\xi)}{\zeta+\xi}
\end{gathered}
$$

we can express $w_{A B A B}$ in terms of the homogeneous disk amplitudes. It contains 10 unknown weight constants. They can be decided by requiring symmetries of the amplitude. Eq. (3.7) already has the symmetry under interchanging $A_{i}$ with $B_{i}$. We further require the symmetry under $\left(A_{1} \leftrightarrow A_{2}\right)$ and that under $\left(B_{1} \leftrightarrow B_{2}\right)$. The unknown constants are determined up to overall normalization. The weights $\left(a_{1}, a_{2}, a_{4}, a_{6}, b_{2}\right)$ have the same non-zero value and the remaining constants equal to zero.

In this way, $w_{A B A B}$ is determined up to the normalization constant. When we choose the normalization constant properly $\left(a_{1}=1\right)$, the obtained amplitude indeed coincides with that obtained from the Schwinger-Dyson equations in section 2 f.

\section{Discussion}

In the previous section we derived the heterogeneous disk amplitude $w_{A B A B}$ only through geometrical consideration. We required the symmetries of the amplitude there, however, we consider that the rules of splitting and the consistency with $w_{A B}$ were essential.

Let us discuss to what extent we can generalize the result we obtained. According to the rules of loop splitting, we can expect that $w_{A B}$ consists of three terms ${ }^{5}($ see Fig. 2). The rules, however, do not give the information on the relative weight of each term. That is, the weight of the last term of the numerator of Eq. (3.9) relative to the first two terms is unknown. When we treat this weight as an additional unknown constant, we can also determine it in the same way to section 3 . This shows that we can also derive $w_{A B}$ from the rules of loop splitting and the consistency between $w_{A B}$ and $w_{A B A B}$. From this fact, we speculate that any heterogeneous

\footnotetext{
${ }^{2}$ In a similar manner we can also reproduce the terms proportional to $t^{4 / 3}$ in Eq. (2.16).

${ }^{3}$ Here we neglect the term proportional to $t^{4 / 3}$.
} 
loop amplitudes can be determined from the loop-splitting rules and the consistency among the relevant amplitudes.

In boundary conformal field theory, the boundary operator $\sigma$ plays the role to make discontinuity in matter state on the boundary. As we saw in section 3, when it is dressed with gravity, it also makes a discontinuity in the geometrical state of the boundary. Here let us comment on the relation with the boundary operators [15, 16] which appear in the scaling operators in the matrix models. In Ref. [16] it is argued that the scaling operator $\hat{\sigma}_{n(m+1)}=\hat{\mathcal{B}}_{n}$ has the effect to split a loop into $k$ loops in the case of $(m, m+1)$ model, where $k \leq n$. When two $\sigma$ approach each other, one can consider that they change the spin state on the boundary locally, and they have the effect to split a loop into two loops [9, 12]. The boundary operator $\hat{B}_{2}$ can be identified with the operator obtained in the limit where two $\sigma$ approach each other. We expect that $\hat{B}_{n}$ can be constructed by making a linear combination of the operators obtained when $2(k-1)$ spin operators $\sigma$ get together, where $1 \leq k \leq n$.

\section{Acknowledgements}

We would like to express our gratitude to Professor I. K. Kostov, because this research has started from the discussion with him. We are also grateful to Professor M. Ninomiya for warmhearted encouragement. Thanks are also due to members of YITP, where one of the authors (A.I.) stayed several times during the completion of this work.

\section{Appendix A Inverse Laplace transformation}

We summarize here some useful relations on inverse Laplace transformation. The Laplace transformation of a function $f\left(\ell_{i}\right)$ is defined by the relation

$$
\mathcal{L}_{i}\left[f\left(\ell_{i}\right)\right]=F\left(\zeta_{i}\right)=\int_{0}^{\infty} d \ell_{i} e^{-\ell_{i} \zeta_{i}} f\left(\ell_{i}\right)
$$

The following three relations are useful in calculating the inverse Laplace transformation,

$$
\begin{aligned}
& \mathcal{L}_{1}^{-1} \mathcal{L}_{2}^{-1}\left[\frac{1}{\zeta_{1}+\zeta_{2}}\right]=\delta\left(\ell_{1}-\ell_{2}\right), \\
& \mathcal{L}_{1}^{-1} \mathcal{L}_{2}^{-1}\left[\frac{F\left(\zeta_{1}\right)}{\zeta_{1}+\zeta_{2}}\right]=\theta\left(\ell_{1}-\ell_{2}\right) f\left(\ell_{1}-\ell_{2}\right), \\
& \mathcal{L}_{i}^{-1}\left[F\left(\zeta_{i}\right) G\left(\zeta_{i}\right)\right]=\int_{0}^{\ell_{i}} d \ell^{\prime} f\left(\ell^{\prime}\right) g\left(\ell_{i}-\ell^{\prime}\right)=f\left(\ell_{i}\right) *_{i} g\left(\ell_{i}\right) .
\end{aligned}
$$

Here $F\left(\zeta_{i}\right)$ and $G\left(\zeta_{j}\right)$ represent the Laplace transformed functions of $f\left(\ell_{i}\right)$ and $g\left(\ell_{j}\right)$ respectively, and the symbol $*_{i}$ in the third equation denotes the convolution with respect to $\ell_{i}$. All of the inverse Laplace transformations of the amplitudes in section 3 can be performed by combining these relations.

\footnotetext{
${ }^{4}$ Eq. (A.3) can be derived combining Eqs. (A.2) and A.4).
} 
As examples, we show the inverse Laplace transformations of some terms in Eq. (3.3) in the following:

$$
\begin{gathered}
\mathcal{L}_{A_{1}}^{-1} \mathcal{L}_{A_{2}}^{-1} \mathcal{L}_{B_{1}}^{-1} \mathcal{L}_{B_{2}}^{-1}\left[\frac{w_{A A B}\left(\zeta_{A_{1}}, \zeta_{A_{2}}, \zeta_{B_{1}}\right) w\left(\zeta_{B_{2}}\right)}{\zeta_{A_{2}}+\zeta_{B_{2}}}\right] \\
=\left[\theta\left(\ell_{A_{2}}-\ell_{B_{2}}\right) \mathcal{W}_{A A B}\left(\ell_{A_{1}}, \ell_{A_{2}}-\ell_{B_{2}}, \ell_{B_{1}}\right)\right] *_{B_{2}} \mathcal{W}\left(\ell_{B_{2}}\right) \\
=\int_{0}^{\min \left(\ell_{A_{2}}, \ell_{B_{2}}\right)} d \ell^{\prime} \mathcal{W}_{A A B}\left(\ell_{A_{1}}, \ell_{A_{2}}-\ell^{\prime}, \ell_{B_{1}}\right) \mathcal{W}\left(\ell_{B_{2}}-\ell^{\prime}\right), \\
\mathcal{L}_{A_{1}}^{-1} \mathcal{L}_{A_{2}}^{-1} \mathcal{L}_{B_{1}}^{-1} \mathcal{L}_{B_{2}}^{-1}\left[\frac{w\left(\zeta_{A_{1}}\right) w\left(\zeta_{A_{2}}\right) w\left(\zeta_{B_{2}}\right)}{\left(\zeta_{A_{1}}+\zeta_{B_{2}}\right)\left(\zeta_{A_{2}}+\zeta_{B_{1}}\right)\left(\zeta_{A_{2}}+\zeta_{B_{2}}\right)}\right] \\
=\left[\theta\left(\ell_{A_{1}}-\ell_{B_{2}}\right) \mathcal{W}\left(\ell_{A_{1}}-\ell_{B_{2}}\right)\right] \\
*_{B_{2}}\left\{\left[\theta\left(\ell_{A_{2}}-\ell_{B_{1}}\right) \mathcal{W}\left(\ell_{A_{2}}-\ell_{B_{1}}\right)\right] *_{A_{2}}\left[\theta\left(\ell_{B_{2}}-\ell_{A_{2}}\right) \mathcal{W}\left(\ell_{B_{2}}-\ell_{A_{2}}\right)\right]\right\} .
\end{gathered}
$$

From Eq. (A.5), one finds that this term corresponds to the first diagram in Fig. : 5. Similarly the amplitude (A.6) can be represented by Fig. 11 . 


\section{References}

[1] Harish-Chandra, Amer. J. Math. 79 (1957), 87.

C. Itzykson and J.-B. Zuber, J. Math. Phys. 21 (1980), 411.

[2] V. A. Kazakov, Phys. Lett. A119 (1986), 140.

D. Boulatov and V. A. Kazakov, Phys. Lett. B186 (1987), 379.

[3] M. Douglas, "The Two-matrix Model", Proceedings of Cargese Workshop, 1990.

T. Tada, Phys. Lett. B259 (1991), 442.

[4] T. Tada and M. Yamaguchi, Phys. Lett. B250 (1990), 38.

[5] J.-M. Daul, V. A. Kazakov and I. K. Kostov, Nucl. Phys. B409 (1993), 311.

[6] V.Pasquier, Nucl. Phys. B285 (1987), 162; J. Phys. 20 (1987), 5707.

[7] E. Gava and K. S. Narain, Phys. Lett. B263 (1991) 213.

[8] M. Staudacher, Phys. Lett. B305 (1993), 332.

[9] M. Ikehara, N. Ishibashi, H. Kawai, T. Mogami, R. Nakayama and N. Sasakura, Phys. Rev. D50 (1994) 7467.

[10] F. Sugino and T. Yoneya, Phys. Rev. D53 (1996), 4448.

[11] M. Anazawa, A. Ishikawa and H. Tanaka, Nucl. Phys. B514 (1998), 419.

[12] M. Anazawa and A. Ishikawa, Prog. Theor. Phys. 104 (2000), 1309.

[13] G. Moore, N. Seiberg and M. Staudacher, Nucl. Phys. B362 (1991), 665.

[14] J. L. Cardy, Nucl. Phys. B324 (1989), 581.

[15] E. Martinec, G. Moore and N. Seiberg, Phys. Lett. B263 (1991), 190.

[16] M. Anazawa, Nucl. Phys. B501 (1997), 251. 


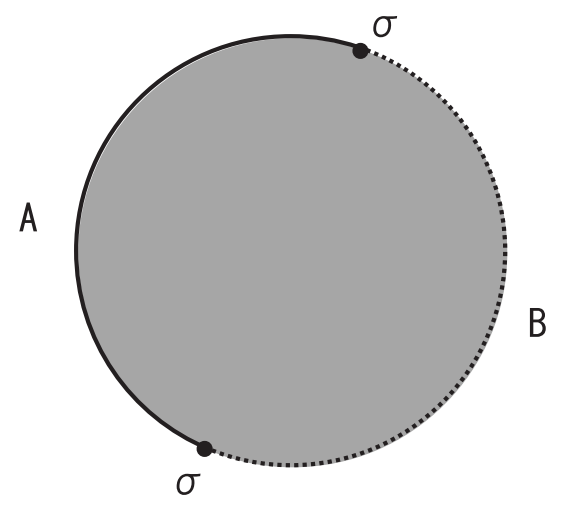

Figure 1: The two spin operators $\sigma$ make discontinuities in the matter configuration on the boundary.
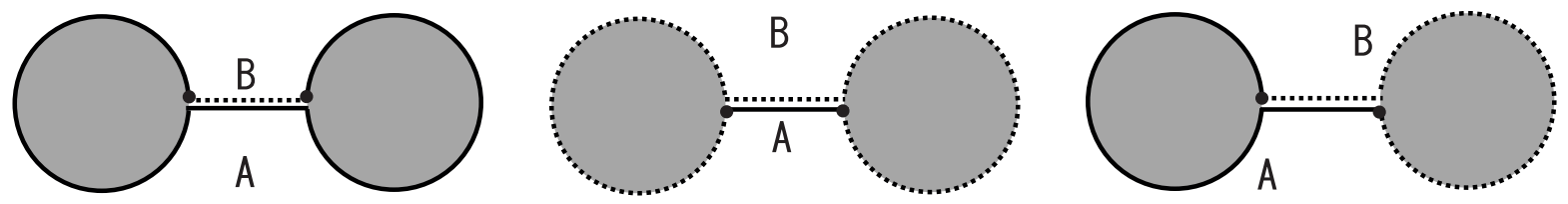

Figure 2: The diagrams representing the first three terms in Eq. (3.2) 


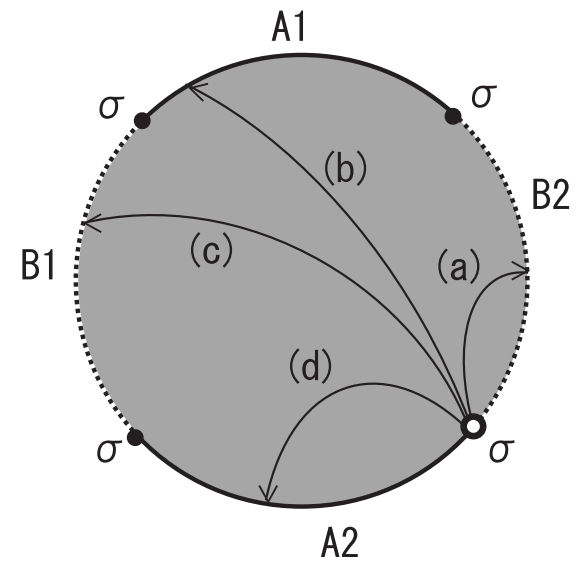

Figure 3: There are four cases depending on the behavior of the spin operator we focus.

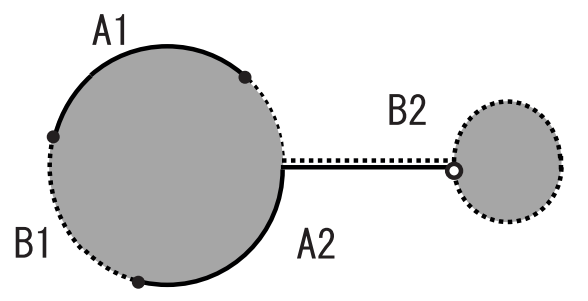

Figure 4: This shows the intermediate situation corresponding to the case (a). Observe that the spin operator we focus is situated at the right end of the double line.

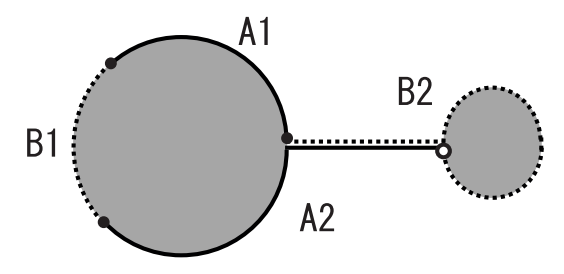

$(\mathrm{a}-1)$

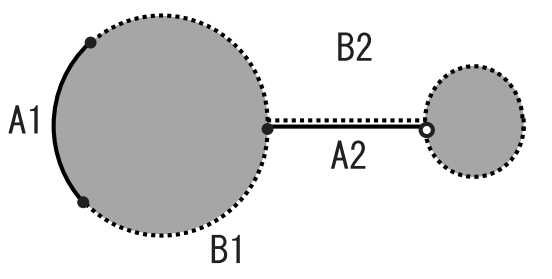

$(\mathrm{a}-2)$

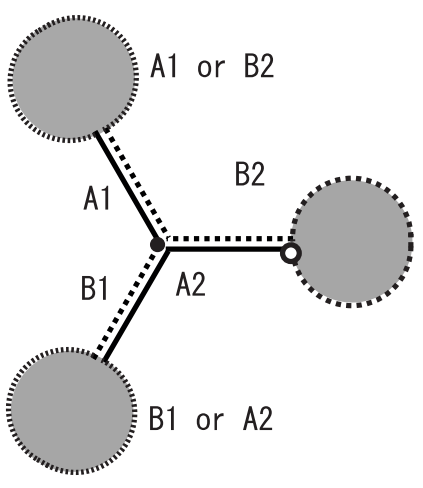

$(\mathrm{a}-3)$

Figure 5: The case (a) is furthermore classified into the three sub cases.

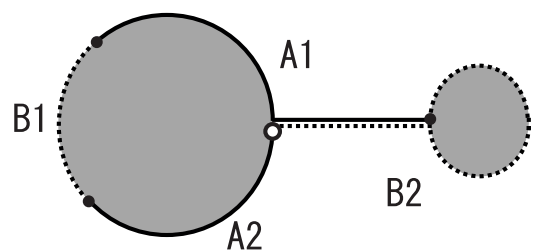

$(\mathrm{b}-1)$

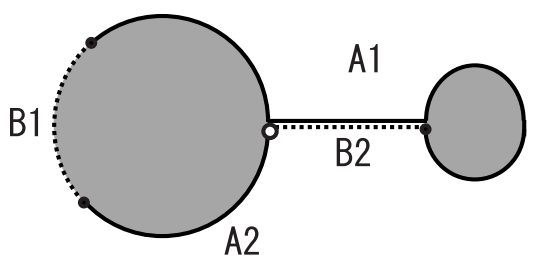

(b-2)

Figure 6: The case (b) is also classified into the two sub cases. 


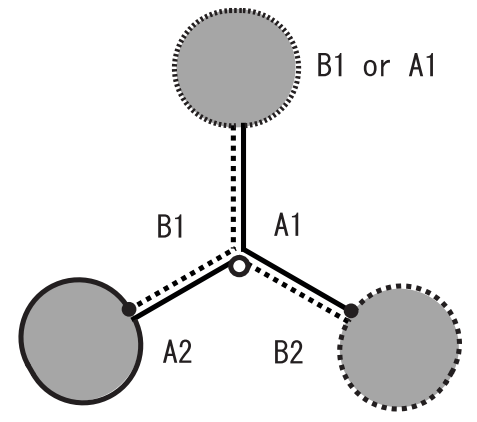

Figure 7: The doubly counted diagram between (b-1) and (c-1)

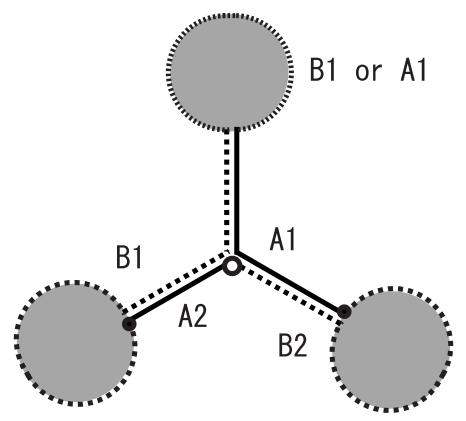

Figure 9: The doubly counted diagram between (b-1) and (c-2)

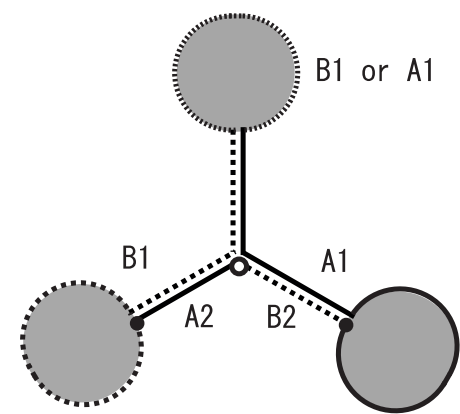

Figure 8: The doubly counted diagram between (b-2) and (c-2)

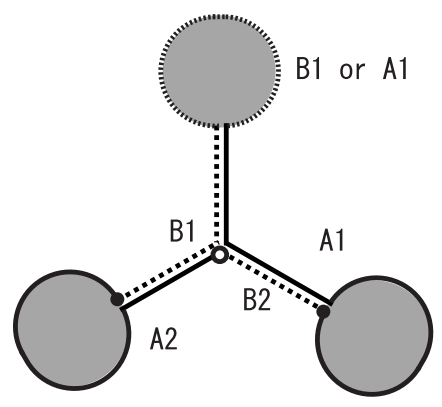

Figure 10: The doubly counted diagram between (b-2) and (c-1)

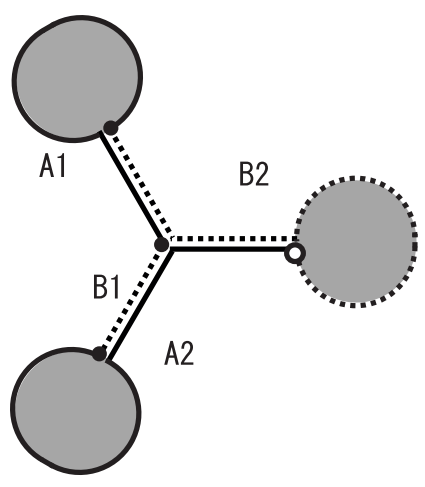

Figure 11: The diagram corresponding to Eq. (A.6) 